\title{
Confinamiento y su incidencia en las relaciones familiares de la ciudadela San José de la parroquia 12 de marzo del cantón Portoviejo
}

\author{
Confinement and its impact on family relationships in the "Ciudadela San José, Parroquia 12 \\ de Marzo, Cantón Portoviejo"
}

Xavier Alexis Macías Moreira' (id (8), Vicenta Inmaculada Ramona Aveiga Macay²

\section{Cómo citar}

Macías, X. y Aveiga, V. (2021). Confinamiento y su incidencia en las relaciones familiares de la ciudadela San José de la parroquia 12 de marzo del cantón Portoviejo. Socialium, 5(2), 120-139. https://doi.org/10.26490/uncp.sl.2021.5.2.931

${ }^{1}$ Egresado de la Carrera de Trabajo Social de la Facultad de Ciencias Humanísticas y Sociales.

Universidad Técnica de Manabí, Portoviejo, Ecuador.

xmacias0653@utm.edu.ec

$2 \mathrm{PhD}$ en Ciencias Pedagógicas, Magister en Gerencia Educativa

Profesional, Licenciada en Servicio Social. Universidad Técnica de Manabí, Portoviejo, Ecuador. vicenta.aveiga@utm.edu.ec

Arbitrado por pares ciegos Recibido: 02/05/2021 Aceptado: $29 / 06 / 2021$

\section{RESUMEN}

Las familias han atravesado por una serie de cambios y adaptaciones durante el confinamiento por la pandemia de la COVID-19, por lo que el objetivo de este estudio fue analizar el confinamiento y su influencia en las relaciones familiares de la ciudadela "San José" de la parroquia 12 de marzo del cantón Portoviejo. El desarrollo de esta investigación se fundamentó en un estudio de tipo descriptivo, con enfoque cuantitativo, para la obtención de información relevante del tema objeto de estudio se realizó una revisión bibliográfica, a nivel empírico se empleó la técnica de la encuesta y se utilizó como instrumento un cuestionario de 10 preguntas, la muestra fue aleatoria intencional con un total de 30 familias que residen en la ciudadela "San José". Los resultados evidenciaron la realidad en la que se sitúan las familias de como impactó en cada miembro del hogar y de los cambios que han tenido que realizar durante el confinamiento por la pandemia del COVID-19 para así adaptarse a esta nueva realidad, en conclusión, el sistema familiar afrontó ciertas adversidades y problemas sociales que afectaron e influyeron en la convivencia con sus miembros, en algunos casos fue positivo, debido a que se realizaron modificaciones para ajustarse al contexto en el que vivían las familias fortaleciéndose los vínculos mejorando sus relaciones familiares, en otros, el confinamiento provocó inestabilidad en la convivencia, debido a que surgieron problemas para superar las calamidades que existían en su entorno.

Palabras clave: familia; confinado; convivencia; relaciones familiares.

\section{ABSTRACT}

Families have lived a series of changes and adaptations during confinement by the COVID-19 pandemic, so the objective of this study was analyze the confinement and its influence on families from Ciudadela "San José"- Parroquia 12 de marzo- Canton Portoviejo. The development of this research was based on a descriptive study, with a quantitative focus, to obtain relevant information on the topic study. It used a bibliographic review with an empirical level, the survey technique was made and 10-questions was used as an instrument. The sample was random with a total of 30 families from "San José" citadel. The results showed the reality in which the families are situated of how it impacted each member of the household and the changes they have had to make during confinement due to the COVID-19 pandemic in order to adapt to this new reality, in conclusion, The family system faced certain adversities and social problems that affected and influenced the coexistence with its members, in some cases it was positive, because modifications were made to adjust to the context in which the families lived, strengthening ties by improving their family relationships, in others, the confinement caused instability in the coexistence, due to the fact that problems arose to overcome the calamities that existed in their environment.

Keywords: family; confined; coexistence; family relationships. 


\section{Introducción}

La pandemia relacionada a la COVID-19 es una enfermedad que ha traído consigo muchos cambios en el estilo de vida de las personas, lo cual ha ocasionado un proceso de aislamiento social como medida de protección para evitar contagios y esto a su vez otorgó la oportunidad de conocer la dinámica que existe dentro del hogar. Desde EFE/ Redacción Primicias (2020) subraya lo expresado por el jefe de la OMS, Hans Kluje exponiendo que, "el aislamiento, el distanciamiento físico, el cierre de escuelas y lugares de trabajo, son desafíos que nos afectan, y es natural sentir estrés, ansiedad, miedo y soledad en estos momentos" (párr. 2).

En este sentido por medio de una entrevista que estableció la prensa El Telégrafo (2020), la socióloga Paola Sánchez de la Fundación Ahínco, afirma que la emergencia sanitaria ha cambiado la normalidad de la interacción familiar debido a que el tiempo en actividades familiares se ha extendido y no ya solo a los fines de semana como anteriormente sucedía.

De forma parecida Montero, et al. (2020) especifica que el confinamiento ha provocado que las familias realicen cambios en el estilo que inciden no tan solo en la dinámica familiar, sino también en lo laboral y social, por lo que, al interno de las familias, se ha desplegado nuevos modos de convivencia, roles y retos que beneficien la convivencia en días de confinamiento. De este modo Soberanis (2020) ha visto la contingencia de la COVID-19 un escenario de convivencia forzada, debido a que la casa ha significado el lugar de trabajo para los padres, el lugar de estudio para los hijos e incluso el lugar de convivencia social con otras personas a través de la tecnología.

Hay que tener en claro que la situación de confinamiento en una pandemia, es una situación fuera de lo que la persona considera normal, es así que Comunicación La Salle Paterna (2020) expresa que, en el contexto de la pandemia, las personas generan estados emocionales que se deben aprender a gestionar de manera correcta para mantener un ambiente familiar con buenas relaciones, por lo que se debe intentar reducir el estado de ánimo negativo que puede producir un confinamiento y fortalecer un ambiente positivo.

A esto, la psicóloga Berzosa (2020) añade que "las parejas han tenido que convivir día y noche sin poder escaparse a realizar sus actividades diarias aumentando así el tiempo de exposición a lo cotidiano en una situación compleja, cargada de incertidumbre, estrés, compaginando ese tiempo con los hijos" (párr. 3). Por lo que se evidencia el reto que esto puede significar para las familias.

Ahora bien, las personas que componen una familia tiene como principal característica mantener vínculos afectivos entre sí, lo que hace que se sientan seguros, tranquilos, estables, etc. Sin embargo, también existen vínculos que generan inseguridad, inestabilidad o miedo, y en un contexto de confinamiento estos 
vínculos pueden percibirse o expresarse de manera más intensa entre los miembros del hogar, dando lugar a modos de relación diferentes a los que ya estaban establecidos (Fundación Lluís Alcanyís, 2020). En esta línea, el Doctor en psicología Poblete (2020) indica que "las diversas restricciones impuestas sobre nuestro comportamiento y el aumento en la frecuencia de las interacciones familiares que se ha suscitado a partir del confinamiento son factores que evidentemente propician el surgimiento de tensión" (párr. 24).

Para el Centro de Apoyo a las familias del Ayuntamiento de Madrid (2020) indica que la situación de la pandemia ha dado paso a que algunas familias pasen por un contexto de crisis ocasionado por el confinamiento, la propagación del virus, la información cotidiana referente a la pandemia, las tareas laborales o académicas, las medidas de higiene, la observación del estado de salud propio, familiar y colectivo y esto inevitablemente conlleva a estos cognitivos como la preocupación, ansiedad estrés, entre otros.

En este sentido Concha (2020), expresa que las relaciones afectivas en el entorno familiar pueden apreciarse de forma más intensa, dando lugar a diferentes maneras de relación y, como probablemente en ninguna de las situaciones conocidas hasta el momento ha existido una convivencia tan intensa entre los miembros que habitan un hogar, es un escenario que necesita un proceso de aprendizaje y adaptación para así poder sobrellevarla de la mejor forma.

De acuerdo al Centro de Apoyo a las Familias del Ayuntamiento de Madrid (2020) el afrontamiento de situaciones de crisis conlleva de forma común a una repuesta adaptativa por parte de las familias a través de una adecuada gestión que requiere conocimientos, organización, comunicación, cambios y medidas de afrontamiento que realizadas en conjunto fortalecen la dinámica familiar, es así que el sistema familiar es imprescindible para afrontar adversidades como lo es la interrupción drástica de contacto con otras personas, que en tiempos de pandemia ha pasado a formar parte de una realidad virtual de los individuos, recobrando de manera intensiva la unión familiar.

El nuevo escenario que impone la pandemia ha representado un cambio de rutina cotidiana lo que implica cambios en la dinámica familiar desplegando los recursos que se poseen e incorporar otros para manejar la interacción familiar que supone permanecer en confinamiento y hacerlo de forma correcta donde pueda existir una convivencia adecuada, así lo afirman (Ibarra y Pérez 2020).

La organización internacional Aldeas Infantiles SOS España (2020) señala que la cuarentena ha establecido nuevas formas de relacionarse en pareja, en familia, entre padres e hijos, y también entre hermanos. La rivalidad y el conflicto, que son parte de las relaciones fraternales, se acentúan, pero también lo hacen el apego y la complicidad. Entendiéndose a este punto como una realidad diversa; la intensidad emocional 
en los días de confinamiento no solo aumenta la probabilidad de que surjan nuevos problemas o discusiones fraternales, sino que también favorece la aparición de conductas positivas y la generación de vínculos más estrechos.

El Centro de Apoyo a las Familias del Ayuntamiento de Madrid (2020) indica que, en momentos de confinamiento, la convivencia familiar se convierte en el día a día, lo que permite que, en un entorno familiar positivo, los valores que, habitualmente se inculcan a sus familiares a través de verbalizaciones, tengan mayor efectividad en las acciones y tiempo que se pasa juntos.

Considerando que la familia es la primera institución de la sociedad y la más importante debido a que es fundamental para el desarrollo psicosocial de las personas, se indica que la conducta del mismo depende mucho de los valores aprendidos en casa y los cuales también son reforzados en el entorno social por amistades o en las instituciones educativas (García, 2020).

Reforzando los efectos positivos que puede traer el confinamiento en las relaciones familiares, Cabrera et al. (2020) realizó una investigación en las familias colombianas, demostrando que "las relaciones internas en la familia durante el confinamiento, el 29,28 \% de la población encuestada piensa que hay discusiones con mayor frecuencia en su familia, mientras que el $84,41 \%$ que hay mayores muestras de afecto" (p. 14). De forma parecida Castellanos (2020) difundió en la revista familia de Ecuador una encuesta a un número determinado de familias donde se muestra los aspectos positivos del confinamiento, en el que algunas familias han logrado fortalecer sus lazos gracias al tiempo que pasaron juntos. Las relaciones entre madres e hijas fueron las que más se beneficiaron del tiempo en cuarentena, por lo que la autora antes mencionada especifica que estas relaciones fueron las que más se fortalecieron en el confinamiento, por el cual el $60 \%$ de familias encuestadas aseguraron que el vínculo entre estos dos miembros del hogar era mucho mayor. También enfatizó que este escenario requiere la utilización que permitan a las familias adaptarse a la nueva realidad y que se promueva el desarrollo de la resiliencia y la salud emocional familiar.

Otros estudios favorables que realizó la profesional en Trabajo Social a familias ecuatorianas demuestran que, los elementos positivos de la convivencia familiar, que constantemente identifican las familias, se relacionan con comprender y valorar las prácticas de diálogo, tolerancia y en especial las prácticas religiosas, como referentes de códigos de conducta, de orden y de brindar tranquilidad frente a la incertidumbre que se vivía (Molina, 2020).

En este sentido, Olmos (2017) indica que en la familia se va construyendo la personalidad de cada uno de sus miembros convirtiéndose en el pilar sobre el cual se fundamenta el desarrollo psicológico, social y físico del ser humano, por lo que al crecer en un buen entorno familiar la sociedad se enfrentaría a menos 
problemáticas sociales debido a que se tendría mejor definido el sentido de responsabilidad, habría menos violencia tanto dentro como fuera del hogar, además habría más respeto tanto para la naturaleza como para el entorno social, evitando de esta manera otros conflictos sociales. Pero si el núcleo familiar es disfuncional la sociedad padece de estas fracturas y las refleja en sus relaciones y entornos; surgen acciones tales como la violencia ya sea física verbal o emocional, desapegos e inconformidades.

De esta forma Soberanis (2020) argumenta que al inicio de la cuarentena existían expectativas positivas de las actividades que se podrían realizar al tener más tiempo y pasar momentos de calidad en familia, como jugar y estudiar con los hijos e incluso convivir en pareja. Sin embargo, a medida que ha transcurrido el confinamiento, se convirtió en una situación cada vez más difícil de manejar para padres e hijos que se desenvolvían en un no muy buen entorno familiar y que no estaban acostumbrado a pasar mucho tiempo juntos, perdiendo de esta manera el equilibrio familiar, la disciplina, los hábitos y las actividades.

Es así que la prensa digital El Telégrafo (2020) compartió un comunicado de la OMS en el que emite una alerta de que el confinamiento también tiene el potencial de exacerbar las interacciones familiares negativas y disfuncionales, sobre todo cuando estas ya existían previamente convirtiéndose así en una situación más difícil de controlar durante una convivencia más prolongada. Además, argumenta que la pandemia por la COVID-19 está causando un aumento de problemas sociales como lo son la violencia doméstica, el abuso sexual y el abuso infantil.

En este punto se resaltan cifras en Ecuador sobre el aumento de la violencia en el contexto del confinamiento, indicando que, en los primeros meses de la pandemia, específicamente hasta el mes de abril del 2020 los casos de violencia aumentaron un 47\%, recibiendo alrededor de 587 llamadas especialmente de mujeres pidiendo ayuda frente a agresores que violentan contra su integridad física y psicológica. Este tema es grave por motivo de que, al estar en sus hogares en horarios de salidas no permitidas, las personas no tienen otra opción que permanecer en sus casas junto a sus victimarios (Montero, et al. 2020).

La prensa digital El Telégrafo (2020), comparte información complementaria con lo antes mencionado e indica que el director general de Sistema Integrado de Seguridad ECU-911, Juan Zapata, manifestó que, desde el inicio de la crisis sanitaria, es decir desde el 12 de marzo hasta noviembre del 2020, la institución que preside, a escala nacional, registra 70,439 casos de violencia intrafamiliar.

Complementando lo antes mencionado, la World Vision (2020) manifiesta que, la tensión y el desequilibrio emocional se convierten en factores desfavorables de la violencia doméstica. Este reporte ratifica que el maltrato infantil durante el 2020 escaló de un 20\% a un 32\%, estos datos representan cerca 
de unos 85 millones de niños a nivel mundial se encuentran expuestos a abusos físicos, psicológicos, emocionales e incluso sexuales.

La socióloga, de la fundación Ahínco expresa que, el tema de la familia hay que tratarlo desde una perspectiva de clase, motivado a la desigual situación económica que viven los países. La experta expuso que las familias de escasos recursos económicos durante el confinamiento ocasionado por la pandemia nunca dejaron de acudir a las calles a trabajar, siendo sus ingresos pudiendo ponderar una situación de violencia intrafamiliar, debido a sentimientos de frustración al no poder solventar sus gastos y desahogándose con los menores, originando de tal manera este tipo de problema social (El Telégrafo, 2020).

Lo mencionado anteriormente concuerda con Cabrera et al. (2020) e indica que "algunos han perdido el trabajo por recortes de personal debido que la situación obligó a algunas empresas tomar estas medidas, la ayuda externa para el mantenimiento del hogar desapareció, lo que generó aumento de la tensión en las relaciones familiares" (p. 2). Por otro lado, el autor antes mencionado indica que es necesario precisar que, a raíz de diversos cambios, las familias han tenido que modificar sus actividades, en la encuesta realizada a las familias colombianas el $49,12 \%$ de los participantes, a partir de la cuarentena se ha modificado la distribución de tareas del hogar, esto ocurre para $13.90 \%$ de los hombres y para el 35,22 \% de las mujeres. El porcentaje mayor en las mujeres se debe a que ellas tradicionalmente han asumido mayores responsabilidades en las tareas de cuidado de los miembros de la familia y del mantenimiento del hogar.

Es así que en las relaciones parentales Cabrera et al. (2020) el confinamiento cambió el desarrollo de las actividades diarias por lo que los padres tuvieron que asumir de manera permanente el acompañamiento escolar de sus hijos y en muchos casos ser profesores de los niños más pequeños, función a la cual estaban acostumbrado a destinárselo al docente de determinada institución educativa. De esta forma la educación de los hijos desde el hogar ha significado un gran reto para las familias debido a que tenían que ejecutar en un solo espacio físico roles de crianza, realización de actividades del hogar y desempeñar funciones labores, por lo que en ciertos padres resultó un desborde emocional para llevar a cabo dichos roles (MezaTorres y Ramírez-Ramírez, 2020).

En la Universidad Nacional de Colombia, la Agencia de Noticias UN (2020), coincide con lo antes mencionado expresando a lo largo de las generaciones, los padres encargan la responsabilidad de la educación y el seguimiento escolar en las instituciones educativas. Sin embargo, en el contexto actual, los padres han tenido que estar inmersos en el proceso instructivo y académico de sus hijos, lo que puede generar un reto en la capacidad de manejar la atención que los hijos requieren. 
La Facultad de Psicología de la Universidad de Buenos Aires (2020) a través del Observatorio de Psicología Social Aplicada realizó investigaciones que corroboran lo antes mencionado y en sus estudios se demuestra que en su población encuestada el 56 \% de padres de familia indicaron la modalidad de educación virtual ha resultado muy dificultosa. Por otra parte, el 30 \% señala que el hecho de tener a los hijos en el hogar realizando sus tareas escolares ha producido un deterioro de la dinámica de las relaciones familiares. Lo expresado anteriormente completa con la percepción que tienen los padres respecto al estado de ánimo que presentan sus hijos como consecuencia de la escolaridad virtual, describiendo estados emocionales de sus hijos como lo son: cansancio, ansiedad, aburrimiento, tristeza, angustia, preocupación, desánimo y depresión.

Es necesario mencionar lo indicado por Nivela, et al. (2020) "la educación en el hogar supone la discriminación y acentuación de brechas y desigualdades existentes, entre quienes pueden tener acceso a la educación virtual porque poseen en sus hogares, acceso a internet, computadora, teléfonos inteligentes, y los que no" (p. 28).

Resulta indudable que el orden familiar y del mundo han sido alterados por lo que, como consecuencia, la mente de las personas también se ha visto afectada, provocando en muchas ocasiones malestar, irritación o ansiedad. Es así que dicho desorden facilita el surgimiento de conflictos entre quienes conviven en un mismo hogar, impactando directamente sobre la atmósfera relacional de la familia (Poblete, 2020). Así se puede decir que la casa, epicentro del confinamiento, además de espacio físico deviene espacio de la familia, donde se resguarda la intimidad, se intercambian las responsabilidades de la vida cotidiana que tiene cada miembro de la familia y se manifiestan los afectos, los vínculos; de igual forma lo hace la limitación de relaciones interpersonales con los familiares y amigos y de interacción con el entorno, por lo que se forman emociones negativas, ansiedad, miedo, a la par, que la situación del confinamiento representa una oportunidad para el reencuentro consigo mismo y con la familia. La realidad del aislamiento, delimitará un antes y un después en diferentes esferas, dentro de las cuales está involucrado la convivencia familiar (Ibarra y Pérez, 2020).

Por lo tanto, el estudio de esta problemática está apoyado en un modelo sistémico-ecológico por cuanto se analiza la interacción familiar y la comunicación que existe entre los integrantes de una familia y como el confinamiento causado por la pandemia de la COVID-19 ha incidido en los integrantes de una familia y a la vez en la dinámica familiar, por lo que se aborda los cambios que han tenido que realizar las familias para adaptarse a una nueva realidad y de las adversidades que pudieron suscitarse durante el confinamiento, así mismo de los problemas sociales que se han originado en ese ámbito y que ha influido 
en la interacción familiar, por lo que el objetivo de este estudio es analizar el confinamiento y su incidencia en las relaciones familiares de la ciudadela San José de la parroquia 12 de marzo del cantón Portoviejo.

\section{Método}

Tipo de estudio. La investigación se realizó a través de un proceso de revisión bibliográfica de distintos artículos científicos, prensa digital y otras fuentes de veracidad los cuales permitieron obtener información relevante relacionada al tema objeto de estudio que de acuerdo a Méndez y Astudillo (2008) "es un proceso mediante el cual se recopila conceptos con el propósito de obtener un conocimiento sistematizado" (p. 16). Se ejecutó una investigación no experimental de tipo descriptivo que de acuerdo al autor Tamayo (1999), “Comprende la descripción, registro, análisis e interpretación de la naturaleza actual, composición o procesos de los fenómenos." En este sentido se efectuó un enfoque cuantitativo que "consiste en la recolección de datos para probar hipótesis con base a la mediación numérica y el análisis estadístico" (Hernández et al. 2014).

Población y muestra. La población objeto de estudio está conformada por 203 familias que residen en el sector de la Ciudadela San José del cantón Portoviejo, en el cual se utilizó un muestreo aleatorio intencional en el que se logró encuestar a 30 familias pertenecientes al sector antes mencionado.

Instrumentos de recolección de datos. Para la recolección de datos se utilizó como instrumento un cuestionario de 10 preguntas relacionadas al tema investigado, que conforme a lo que indica Anguita, et al., (2003) "es un documento que recoge de forma organizada los indicadores de las variables implicadas en el objetivo de la encuesta" (p. 153). De esta forma de acuerdo a la contestación que se le admitió al encuestado, se establecieron preguntas cerradas en el que la persona encuestada debía elegir entre varias opciones.

Procedimientos de la recolección de datos. El procedimiento empleado en el estudio realizado fue la técnica de la encuesta que conforme a lo que expresa Anguita, et al., (2003) "Permite la obtención de datos sobre una gran variedad de temas en el que la información se recoge de modo estandarizado mediante un cuestionario lo que permite hacer comparaciones intragrupales" (p. 143). En este sentido la técnica fue ejecutada de forma online a través del software de administración de encuestas "formularios google" el cual contenía información relacionada al tema y objetivo del estudio, así como también de las preguntas a contestar cada una con sus opciones. La encuesta fue entregada a la población encuestada por medio de un link a través de redes sociales digitales como lo son Whatsapp y Facebook. 
Aspectos éticos. Los aspectos éticos considerados en la investigación, están relacionados a la protección que obtuvo la población encuestada como participantes de este estudio en el que se consideró la invasión a la privacidad y la confidencialidad en referencia a la divulgación de la información otorgada por el participante bajo su consentimiento, es de esta forma que se detalló el anonimato que tendría el encuestado al brindar información en el proceso de la aplicación de la encuesta.

Análisis de datos. El análisis de los datos se lo realizó en base a la información obtenida durante el proceso de recolección de información en el que por medio del programa de Microsoft Excel se tabuló las opciones contestadas por los participantes obteniendo frecuencias, estableciendo porcentajes y realizando un análisis estadístico de las variables. Los datos recopilados a través del uso de los distintos enfoques y técnicas permitieron realizar un análisis de la realidad en la que se sitúa las familias y así conocer los comportamientos de la población en un contexto de confinamiento producto de la pandemia relacionada a la COVID-19.

\section{Resultado}

Para la obtención de los resultados se aplicó una encuesta a 30 familias residentes de la ciudadela "San José" el cual servirá para demostrar como el confinamiento a raíz de la pandemia COVID-19 ha influido en las relaciones familiares. Los resultados de la aplicación de la encuesta responden a un formato establecido de 10 preguntas:

\section{Tabla 1}

Integrante familiar y estructura familiar

\begin{tabular}{lcc}
\hline Integrante familiar & Frecuencia & Porcentaje \\
\hline Abuelo/a & 2 & $6,6 \%$ \\
Mamá & 15 & $50 \%$ \\
$\begin{array}{l}\text { Papá } \\
\text { Hijo/a }\end{array}$ & 2 & $6,6 \%$ \\
$\begin{array}{l}\text { Sobrino } \\
\text { Otro }\end{array}$ & 10 & $33,33 \%$ \\
Total & 0 & $0 \%$ \\
\hline Estructura familiar & 1 & $3,3 \%$ \\
\hline $\begin{array}{l}\text { Familia nuclear (formada por los progenitores y } \\
\text { uno o más hijos) }\end{array}$ & 30 & $100 \%$ \\
$\begin{array}{l}\text { Familia extensa (habitan en la misma casa abuelos, } \\
\text { tíos, primos y otros parientes) }\end{array}$ & 0 & $96,6 \%$ \\
$\begin{array}{l}\text { Familia monoparental (en el que convive un } \\
\text { progenitor con un su hijo) }\end{array}$ & 1 & $0 \%$ \\
$\begin{array}{l}\text { Familia mixta (en el cual uno o ambas miembros de } \\
\text { la actual pareja tiene uno o varios hijos de uniones } \\
\text { anteriores) }\end{array}$ & 0 & $0 \%$ \\
\hline$\quad$ Total & 30 & $100 \%$ \\
\hline
\end{tabular}


Nota. Tomado de Familias residentes de la ciudadela "San José" del cantón Portoviejo provincia de Manabí (2021).

La mayoría de los integrantes de una familia que participaron en la realización de la encuesta corresponde a las madres con un 50 \%, otra participación elevada la tuvieron los hijos con un 33,3 \%. Por lo que se evidencia una mayor predisposición de tiempo en el hogar tanto de la madre como el hijo/a. En un gran porcentaje se identifica que las familias que formaron parte del proceso estadístico corresponden con un 96,6 \% a la conformación de una familia nuclear formada por progenitores y sus hijos; y con un 3,4 \% se encuentra la familia monoparental en la que un progenitor convive con su hijo/a. De esta forma se demuestra que en su mayoría las familias que residen en el sector están formadas tradicionalmente por la unidad básica de una familia.

\section{Tabla 2}

Interacción familiar, cambio de roles, normas, distribución de tareas a partir del confinamiento de la pandemia

\begin{tabular}{lcc}
\hline Alternativas & Frecuencia & Porcentaje \\
\hline Sí & 23 & $76,6 \%$ \\
No & 7 & $24,4 \%$ \\
No opina & 0 & $0 \%$ \\
\hline Total & 30 & $100 \%$ \\
\hline
\end{tabular}

Nota. Tomado de Familias residentes de la ciudadela “San José” del cantón Portoviejo provincia de Manabí (2021).

Las familias encuestadas indicaron con un 76,6 \% que a raíz del confinamiento producido por la pandemia de la COVID-19 han tenido que modificar las normas y distribución de tareas ajustándose en la realidad en la que se encuentran inmersas, mientras que un 24,4 \% manifestó que no hubo un cambio significativo en ese aspecto.

\section{Tabla 3}

Frecuencia de actividades familiares a partir del confinamiento de la pandemia

\begin{tabular}{lcc}
\hline Alternativas & Frecuencia & Porcentaje \\
\hline Muy Frecuentemente & 5 & $16,6 \%$ \\
Frecuentemente & 8 & $26,6 \%$ \\
Ocasionalmente & 12 & $40 \%$ \\
Poco frecuente & 4 & $13,33 \%$ \\
Nunca & 1 & $3,3 \%$ \\
\hline Total & 30 & $100 \%$ \\
\hline
\end{tabular}

Nota. Tomado de Familias residentes de la ciudadela “San José” del cantón Portoviejo provincia de Manabí (2021). 
Al preguntar con qué frecuencia realizan actividades familiares durante el confinamiento producido por la pandemia, en un gran porcentaje del $40 \%$ indico que de manera ocasional efectuaron actividades familiares, con un $26,6 \%$ manifestaron que se da de manera frecuente y con porcentajes menores está muy frecuentemente con el 16,6 \% y poco frecuente con un 13,3\%. Por lo que se evidencia que las actividades en familia no aumentaron de manera significativa por lo que se mantienen de forma ocasional durante el confinamiento.

\section{Tabla 4}

Dificultad en la interacción familiar producidos por el confinamiento a raíz de la pandemia COVID-19 y entre que miembros de la familia se da con mayor constancia

\begin{tabular}{lcc}
\hline Dificultades & Frecuencia & Porcentaje \\
\hline Conflictos (discusiones, desacuerdos, quejas) & 10 & $33,3 \%$ \\
violencia física (golpes) & 0 & $0 \%$ \\
violencia verbal (gritos, insultos) & 0 & $0 \%$ \\
Desapegos (falta de afecto, apoyo e interés) & 0 & $0 \%$ \\
Ruptura familiar & 0 & $0 \%$ \\
Abandono (aislamiento entre miembros del hogar) & 2 & $6,6 \%$ \\
Ninguno & 18 & $60 \%$ \\
\hline Total & 30 & $100 \%$ \\
\hline Pareja de integrantes familiares & & $43,3 \%$ \\
\hline Entre padre y madre & 13 & $6,6 \%$ \\
Entre madre e hijo & 2 & $3,3 \%$ \\
Entre padre e hijo & 1 & $3,3 \%$ \\
Entre padre e hija & 1 & $0 \%$ \\
Entre madre e hija & 0 & $23,3 \%$ \\
Entre hermanos & 7 & $20 \%$ \\
Otros & 6 & $100 \%$ \\
\hline Total & 30 & $\%$ \\
\hline
\end{tabular}

Nota. Tomado de Familias residentes de la ciudadela "San José" del cantón Portoviejo provincia de Manabí (2021).

La pandemia ha traído consigo algunos efectos negativos en la convivencia familiar entre el más común se encuentran los conflictos con un 33,3\% los cuales comprenden discusiones, desacuerdos y quejas entre los miembros de una familia, por otro lado, en muchas familias han asumido el confinamiento de una mejor forma y no ha ocasionado un aumento de factores negativos, correspondiendo así a un porcentaje del $60 \%$ y en un porcentaje mínimo del 6,6\% se ha presentado aislamiento entre los miembros de una familia. En relación a los conflictos que han aumentado en las familias durante el confinamiento se evidencia que se da con mayor frecuencia entre la pareja (madre y padre), correspondiendo a un 43,3\%, 
además entre hermanos también sucede esta situación otorgándole un $23,3 \%$, por lo que se demuestra que los conflictos suscitados durante el confinamiento se dan con mayor frecuencia entre padres y entre hermanos.

\section{Tabla 5}

Estado emocional que ha generado el confinamiento en algún miembro de la familia y su frecuencia en la afectación de la convivencia familiar

\begin{tabular}{|c|c|c|}
\hline Alternativas & Frecuencia & Porcentaje \\
\hline Estrés & 10 & $33,3 \%$ \\
\hline Depresión & 5 & $16,6 \%$ \\
\hline $\begin{array}{l}\text { Soledad debido al aislamiento con el entorno } \\
\text { exterior }\end{array}$ & 0 & $0 \%$ \\
\hline Miedo & 3 & $10 \%$ \\
\hline Aburrimiento & 6 & $20 \%$ \\
\hline Agresividad & 0 & $0 \%$ \\
\hline Mal humor & 1 & $3,3 \%$ \\
\hline Preocupación & 1 & $3,3 \%$ \\
\hline Sensibilidad & 0 & $0 \%$ \\
\hline Irritabilidad & 0 & $0 \%$ \\
\hline Otros & 0 & $0 \%$ \\
\hline Ninguno & 4 & $13,3 \%$ \\
\hline Total & 30 & $100 \%$ \\
\hline \multicolumn{3}{|l|}{ Afectación en la convivencia familiar } \\
\hline Muy Frecuentemente & 3 & $10 \%$ \\
\hline Frecuentemente & 8 & $26,6 \%$ \\
\hline Ocasionalmente & 6 & $20 \%$ \\
\hline Poco frecuente & 8 & $26,6 \%$ \\
\hline Nunca & 5 & $16,6 \%$ \\
\hline Total & 30 & $100 \%$ \\
\hline
\end{tabular}

Nota. Tomado de Familias residentes de la ciudadela "San José" del cantón Portoviejo provincia de Manabí (2021).

El confinamiento producido por la pandemia ha generado entre los miembros de las familias distintos estados emocionales que les ha afectado, entre el más frecuente está el estrés con un 33,3 \%, así mismo la depresión con un 16,6\% y el aburrimiento con un $20 \%$ han estados presentes durante el confinamiento, pero con una frecuencia menor. Al preguntar con qué frecuencia el estado de ánimo ha afectado a la convivencia familiar durante el confinamiento producido por la pandemia, un porcentaje del $26,6 \%$ indico que se da de manera frecuente, sin embargo el mismo porcentaje también manifestó que se da de forma poco frecuente, por lo que se evidencia que hay integrantes de las familias que no permiten que sus 
estados emocionales afecten la interacción familiar, mientras que otros no pueden tener un manejo adecuado de sus emociones y estos influyen en la dinámica familiar.

\section{Tabla 6}

Situaciones que han influido de manera negativa en la convivencia familiar durante el confinamiento

\begin{tabular}{lcc}
\hline Alternativas & Frecuencia & Porcentaje \\
\hline Padres realizando teletrabajo desde su & 3 & $10 \%$ \\
hogar & 9 & $30 \%$ \\
Hijos estudiando desde el hogar & 11 & $36,6 \%$ \\
Desempleo de un miembro de la familia & 7 & $23,3 \%$ \\
Otro & 30 & $100 \%$ \\
\hline Total & & \\
\hline
\end{tabular}

Nota. Tomado de Familias residentes de la ciudadela "San José" del cantón Portoviejo provincia de Manabí (2021).

Respecto a situaciones que han afectado la convivencia familiar, la población encuestada indico que en su mayoría se debe al desempleo de un miembro de la familia con un $36,6 \%$, mientras que otro factor frecuente es la de los hijos estudiando desde el hogar con un $9 \%$, por lo que se evidencia que el desempleo que actualmente en tiempos de pandemia representa un problema social que ha ido en aumento, ha afectado la dinámica familiar, así mismo la educación de los hijos desde el hogar, pues esto ha representado un cambio en la interacción familiar que no siempre es sobrellevada de la mejor forma.

\section{Tabla 7}

Fortalecimiento de la comunicación y vínculo familiar

\begin{tabular}{lcc}
\hline Alternativas & Frecuencia & Porcentaje \\
\hline Sí & 18 & $60 \%$ \\
No & 12 & $40 \%$ \\
No opina & 0 & $0 \%$ \\
\hline Total & 30 & $100 \%$ \\
\hline
\end{tabular}

Nota. Tomado de Familias residentes de la ciudadela "San José" del cantón Portoviejo provincia de Manabí (2021).

Las respuestas de las familias respecto a la interrogante relacionada a la mejora del vínculo familiar durante el confinamiento, el $60 \%$ de las familias expresaron que obtuvieron un mejor vínculo entre los miembros de su familia, mientras que un 40 \% manifestó que la interacción familiar no mejoro durante el 
confinamiento, por lo que se puede evidenciar que cada familia afronta y vive la realidad del confinamiento de diferentes formas.

\section{Discusión}

Las encuestas realizadas a las familias de la ciudadela "San José" fueron respondidas en su gran mayoría por madres e hijos evidenciándose la disposición de tiempo con la que cuentan en el hogar, debido a que la estructura familiar de los integrantes encuestados corresponde a una estructura nuclear en la que tradicionalmente el padre es el jefe del hogar y cumple con actividades laborales. Este resultado coincide con los datos obtenidos por (Cabrera et al., 2020) en relación a la encuesta realizada a las familias colombianas en el que las responsabilidades del hogar son asumidas mayoritariamente por las mujeres.

Sin embargo, el confinamiento ha ocasionado que la unidad básica de la familia tenga que reestructurar sus normas y tareas dentro del hogar, adaptándose así a la nueva realidad en la que se sitúan, es así que el 76,6 \% de la población encuestada manifestó que fue necesaria un reajuste en las actividades que realizaban dentro del hogar, estos datos corroboran lo mencionado por (Montero, et al., 2020) en el que afirma que el confinamiento, ha producido cambios a lo interno de las familias, por lo que se han visto forzadas a desplegar nuevos modos de convivencia, nuevos roles y retos.

La profesional en Trabajo Social Molina (2020) en los datos que consiguió en su investigación sobre la convivencia familiar en confinamiento obtuvo que entre los principales efectos que el confinamiento generó en la convivencia familiar, se encuentra la calidad de tiempo que han podido, o no, pasar entre sus miembros; reconociendo que este hecho permite la posibilidad de compartir actividades entre todos los integrantes de la familia. Si bien es cierto que el confinamiento ha representado una oportunidad para convivir más en familia y pasar tiempo de calidad, la realidad es que en gran medida de las personas encuestadas específicamente un 40 \% expresaron que las actividades familiares se mantienen de forma ocasional como ocurría antes de que iniciara el confinamiento, por motivo a distintas actividades individuales que tienen que cumplir los integrantes de una familia, sin embargo, un 26,6 \% si han compartido actividades familiares de forma frecuente. Este resultado refleja lo expresado por (Soberanis, 2020) indicando que al principio del confinamiento existían expectativas positivas en cuanto a la relación familiar debido a que se iba a tener mayor tiempo para convivir de forma positiva, pero que en la práctica esto no fue así.

Los conflictos que forman parte de la convivencia con otras personas han tenido un aumento notable a partir del confinamiento en las familias reflejándose en un 33,3 \%, los cuales se da con mayor frecuencia 
entre el padre y la madre. Estos resultados coinciden con lo expresado por (Poblete, 2020) indicando que el confinamiento alteró el orden familiar y como consecuencia el bienestar emocional de las personas se ha visto afectadas ocasionando en muchos casos malestar, tensión, ansiedad e irritación y a su vez estos estados de ánimos han influido en que surjan conflictos dentro del hogar.

De acuerdo a Centro de Apoyo a las Familias del Ayuntamiento de Madrid (2020), aspectos como la reclusión en el hogar, la omnipresencia de la enfermedad, la información cotidiana sobre la pandemia en los medios de comunicación, las conversaciones relacionadas al tema de la pandemia, las medidas sanitarias a seguir y el seguimiento del estado de salud propio, conllevan en muchas personas a un estado cognitivo de estrés y esto hace que la interacción entre los miembros del hogar sea dificultosa, bajo este contexto los datos obtenidos corroboraron con lo antes mencionado debido a que el estrés está presente en los hogares de las familias correspondiendo a un 33,3\% de los encuestados y esto ha representado de manera frecuente $(26,6 \%)$ dificultades en la convivencia familiar.

Otras situaciones que han generado problemas en la interacción familiar con un 36,6 \% de las familias encuestadas, ha sido el desempleo de un miembro de la familia, por lo que a esto se complementa el comentario de la socióloga de la fundación Ahínco, subrayado en El Telégrafo (2020) expresando que la disminución de ingresos económicos incide en la frustración y depresión de los miembros del hogar lo que a su vez puede ocasionar problemas sociales tales como la violencia o actividades ilícitas. Por otro lado, también se encuentra la situación de que los hijos estudien desde el hogar (30 \%) lo cual coincide con los resultados obtenidos por la Facultad de Psicología de la Universidad de Buenos Aires (2020), en una investigación realizada en el observatorio de psicología aplicada, en el que refleja que para el 56 \% de las familias encuestadas manifestaron que tener a sus hijos estudiando desde el hogar ha resultado muy dificultoso y de ese porcentaje un $30 \%$ señala que esa situación produce un deterioro en la dinámica familiar.

A pesar de las adversidades que se han originado a partir del confinamiento en el entorno familiar, a través de diferentes esfuerzos para superar las situaciones se ha visto un vínculo familiar más unido, es así como lo expresan las familias encuestadas, en las que el $60 \%$ indico que el vínculo familiar se fortaleció, sin embargo un 40 \% considero que la comunicación y el vínculo familiar no mejoro, estos datos son muy similares a los obtenidos por (Cabrera et al. 2020) en el que la familia encuestada indicó que se han visto mayores muestras de afecto con un 84.41 \% y el otro 29.28 \% señalo que los conflictos en el hogar aumentaron. 
Es así que se evidencia que el confinamiento es abordado de diferentes formas de acuerdo a la realidad en la que se encuentre la familia, y sobrellevarlo de la mejor forma dependerá de cómo afronten la situación.

\section{Conclusión}

La pandemia de la COVID-19 ha significado un reto para las familias durante el confinamiento, donde la cotidianidad en la que vivían con normas y roles establecidos, se tuvieron que modificar para ajustarse a una nueva realidad y así superar una crisis social y sanitaria, esta situación ha incidido positivamente en las relaciones de algunas familias de la ciudadela San José de la parroquia 12 de marzo del cantón Portoviejo, por cuanto ha permitido compartir más tiempo, realizar actividades, mejorar la interacción entre sus integrantes y fortalecer los vínculos del sistema familiar.

En los casos de las familias con una dinámica deteriorada, se evidenciaron dificultades durante el confinamiento, esto, aumentó conflictos y desacuerdos durante la convivencia, al mismo tiempo, han tenido que lidiar con la propagación de la enfermedad del coronavirus, lo cual ha generado estados de estrés, preocupación, ansiedad, depresión y agresividad, influyendo de forma negativa en las relaciones familiares e incrementando el índice de violencia intrafamiliar.

Otras situaciones problemáticas que ha generado el confinamiento por COVID-19 son el desempleo, aumento de la tasa de pobreza, actividades ilícitas y crisis económica, ocasionando dificultades en las familias para sobrellevar la situación, no obstante, la superación de estas adversidades dependerá de la resiliencia familiar.

\section{Referencias}

Agencia Nacional UN. (2020, 03 de abril). Relaciones familiares a prueba en tiempos de coronavirus. Universidad Nacional de Colombia.

https://agenciadenoticias.unal.edu.co/detalle/article/relaciones-familiares-a-prueba-entiempos-de-coronavirus.html

Aldeas Infantiles SOS España. (2020). Las relaciones entre hermanos en confinamiento: emergencia sanitaria Covid-19.

https://www.observatoriodelainfancia.es/oia/esp/documentos_ficha.aspx?id=7057 
Anguita, J. Labrador, R. y Campos, D. (2003). La encuesta como técnica de investigación. Elaboración de cuestionarios y tratamiento estadístico de los datos (I). Aten Primaria 31(8), 527-538. https://core.ac.uk/download/pdf/82245762.pdf

Berzosa, M. (2020, 12 de agosto). Las relaciones de pareja tras el confinamiento por la Covid-19. The Conversation. https://theconversation.com/las-relaciones-de-pareja-tras-el-confinamiento-porla-covid-19-142857

Cabrera, V., Acuña, L., Docal, M. \& Campos, A. (2020). Vivencias familiares durante el confinamiento por Covid19. Instituto de La Familia - Universidad de La Sabana. https://www.unisabana.edu.co/fileadmin/Archivos_de_usuario/Documentos/instituto-de-lafamilia/Vivencias_familiares_durante_el_confinamiento_por_COVID_19_universidad_de_la_sab ana.pdf

Castellanos, G. (2020, 6 de agosto). La cuarentena mejoró las relaciones entre madres e hijas. Revista Familia. https://www.revistafamilia.ec/padres-e-hijos/cuarentena-mejoro-relaciones-madrese.html

Centro de Apoyo a las Familias del Ayuntamiento de Madrid. (2020). Guía para la convivencia familiar durante la crisis del Covid19. Madrid.es. https://www.madrid.es/UnidadesDescentralizadas/InfanciayFamilia/EntidadesyOrganismos/CAF /EstadoAlarmaGuias/GuiaparalaConvivenciaFamiliarCAF.pdf

Comunicación La Salle Paterna. (2020, 27 de abril). Las relaciones en el ámbito familiar en situación de confinamiento. La Salle Colegio Paterna. https://www.lasallepaterna.es/las-relaciones-en-elambito-familiar-en-situacion-de-confinamiento/

Concha, T. (2020, 10 de abril). El confinamiento afecta a la gestión emocional de la familia y genera conflictos. Agencia EFE. https://www.efe.com/efe/espana/sociedad/el-confinamiento-afecta-ala-gestion-emocional-de-familia-y-genera-conflictos/10004-4218008

EFE/ Redacción Primicias. (2020, 26 de marzo). OMS: el impacto sicológico de Covid-19 no debe ser ignorado. Primicias. https://www.primicias.ec/noticias/sociedad/oms-impacto-psicologicocovid-19-sociedad-ser-ignorado/

El Telégrafo. (2020, 5 de noviembre). 70.439 casos de violencia intrafamiliar se han registrado durante la pandemia. El Telégrafo. https://www.eltelegrafo.com.ec/noticias/actualidad/44/casos-violenciaintrafamiliar-pandemia 
El telégrafo. (2021, 15 de julio). La pandemia por covid-19 también cambio las relaciones familiares. El Telégrafo. https://www.eltelegrafo.com.ec/noticias/sociedad/6/relaciones-familia-covid19

Facultad de Psicología de la Universidad de Buenos Aires, (2020, 5 de octubre). Cuarentena en familia: el $30 \%$ de los argentinos asegura que la dinámica de las relaciones empeoró. Infobae. https://www.infobae.com/tendencias/2020/10/05/cuarentena-en-familia-el-30-de-losargentinos-asegura-que-la-dinamica-de-las-relaciones-empeoro/

Fundación Lluís Alcanyís. (2020). Gestionar situaciones familiares durante el confinamiento. Clínica de Psicología de la Universitat de València. https://www.fundaciolluisalcanyis.org/clinicas/wpcontent/uploads/2020/04/guia-conflictos-clinica-psicologia.pdf

García, M. (2020). Estrés y su influencia en la conducta social de un adolescente en confinamiento por covid-19. [Documento probatorio de examen complexivo de pregrado, Universidad Técnica de Babahoyo] DSpace UTB. http://dspace.utb.edu.ec/bitstream/handle/49000/8964/E-UTB-FCJSEPSCLIN-000362.pdf?sequence=1\&isAllowed $=y$

Hernández, R., Fernández, C. y Baptista, M. (2014). Metodología de la investigación (6ta ed.). McGrawHill.

Ibarra, L. y Pérez, M. (2020). Vivencia de aislamiento y convivencia familiar en los tiempos del Coronavirus. Alternativas Cubanas en Psicología, 8(24). https://www.acupsi.org/articulo/312/vivencia-deaislamiento-y-convivencia-familiar-en-los-tiempos-del-coronavirus.html

Méndez, A. y Astudillo M. (2008). La investigación en la era de la información: guía para realizar la bibliografía y fichas de trabajo. México, Editorial Trillas. http://www.economia.unam.mx/academia/inae/pdf/inae1/u1/5.pdf

Meza-Torres, S. y Ramírez-Ramírez, L. (2020). Ansiedad infantil: estudio exploratorio de la percepción de madres y padres de familia durante el confinamiento de la covid-19 en México. ResearchGate. https://www.researchgate.net/profile/Leticia-Ramirez-

Ramirez/publication/351917797_ANSIEDAD_INFANTIL_ESTUDIO_EXPLORATORIO_DE_LA_PERCE PCION_DE_MADRES_Y_PADRES_DE_FAMILIA_DURANTE_EL_CONFINAMIENTO_DE_LA_COVID19_EN_MEXICO/links/60afd517a6fdcc647ee3202e/ANSIEDAD-INFANTIL-ESTUDIOEXPLORATORIO-DE-LA-PERCEPCION-DE-MADRES-Y-PADRES-DE-FAMILIA-DURANTE-ELCONFINAMIENTO-DE-LA-COVID-19-EN-MEXICO.pdf

Molina, M. (2020). Percepciones de las familias de Socio Vivienda respecto a la convivencia familiar y las condiciones de habitabilidad en situación de confinamiento por COVID -19 [Tesis de pregrado, 
Universidad Católica de Santiago de Guayaquil]. Repositorio institucional UCSG. http://repositorio.ucsg.edu.ec/bitstream/3317/15619/1/T-UCSG-PRE-JUR-TSO-136.pdf

Montero, D., Bolívar, M., Aguirre, L. y Moreno, A. (2020). Violencia intrafamiliar en el marco de la emergencia sanitaria por el Covid-19. CienciAmérica, 9(2), 261-267.

http://cienciamerica.uti.edu.ec/openjournal/index.php/uti/article/view/316/551

Nivela, M., Molina, C. y Campos, R. (2020). El rol de la familia en la educación en casa durante el confinamiento. E-IDEA Journal of Business Sciences, 2(6), 22-29.

https://revista.estudioidea.org/ojs/index.php/eidea/article/view/38/30

Olmos, M. (2017). La familia monoparental y los hábitos de estudio de los estudiantes de quinto año de educación básica de la unidad educativa provincia de Cotopaxi del cantón Pujilí [tesis de pregrado, Universidad Técnica de Ambato]. Repositorio institucional UTA. https://repositorio.uta.edu.ec/bitstream/123456789/26791/1/0503990327\%20MAR\%C3\%8DA \%20ISABEL\%20OLMOS\%20MORENO\%20FINAL.pdf

Poblete, O. (2020, 12 de junio). Reflexión de académico sobre el confinamiento y las emociones en la familia. Universidad de Playa Ancha. https://www.upla.cl/noticias/2020/06/12/reflexion-deacademico-sobre-el-confinamiento-y-las-emociones-en-la-familia/

Soberanis, D. (2020, 14 de mayo). La familia en tiempos de confinamiento. UVM sala de prensa. https://laureate-comunicacion.com/prensa/la-familia-en-tiempos-deconfinamiento/\#.YBSVioPOXIV

Tamayo, M. (1999). Aprender a investigar. Instituto Colombiano Para el Fomento de la Educación Superior ICFES. https://academia.utp.edu.co/grupobasicoclinicayaplicadas/files/2013/06/2.-LaInvestigaci\%c3\%b3n-APRENDER-A-INVESTIGAR-ICFES.pdf

World Vision. (2020). Una tormenta perfecta: más millones de niños corren el riesgo de sufrir violencia durante el confinamiento y la "nueva normalidad". https://www.wvi.org/sites/default/files/202006/COVID-19\%20aftershocks_SP.pdf 


\section{Contribución de los autores}

MMXA: Conceptualización, análisis formal, investigación, redacción: borrador original.

AMVIR: Metodología, supervición, validación, redacción: revisión y edición.

Fuentes de financiamiento.

Autofinanciado

\section{Conflictos de interés}

No presenta conflicto de intereses.

\section{Correspondencia}

xmacias0653@utm.edu.ec 